\title{
Covariance mapping and triple coincidence techniques applied to multielectron dissociative ionization
}

\author{
L J Frasinski, A J Giles, P A Hatherly, J H Posthumus, M R Thompson and K Codling
}

J J Thomson Physical Laboratory, The University of Reading, Whiteknights, Reading, RG6 6AF, United Kingdom.

e-mail: L.J.Frasinski@Reading.ac.uk

Single versus multiphoton multiple ionization of diatomic and triatomic molecules is discussed. The former experiments use synchrotron radiation, while the latter ones require pico- or femtosecond laser pulses focused to an intensity exceeding $10^{14} \mathrm{~W} / \mathrm{cm}^{2}$. A method of improving covariance maps by decorrelating laser fluctuations is presented. Removal of false coincidences in triple coincidence experiments is extended to a varying excitation rate. An explanation of the multielectron dissociative ionization mechanism is sought in terms of bond softening, bond hardening (stabilisation), and ionization enhancement due to Stark-shift excitation. It is expected that this excitation is accompanied by emission of vacuum ultraviolet radiation that differs from high harmonic generation.

\section{Single versus multiphoton ionization}

A molecule can be multiply photoionized using a single, high-energy photon or many, low-energy ones. The former experiments use synchrotron radiation in the vacuum ultraviolet or soft $x$-ray regions of the spectrum, while the latter ones require an intense pico- or femtosecond laser operating usually at visible or near infrared wavelengths.

The multiply ionized molecule dissociates and the fragments gain kinetic energy reflecting the difference between the Coulomb and the binding energies. A time-of-flight (TOF) technique is usually used to obtain the fragment mass spectrum and to measure the kinetic energies and angular distributions. The TOF spectrometer is enclosed in an ultra-high vacuum apparatus. Single photon experiments require good vacuum to avoid contamination of the VUV optics and the synchrotron ring, while in the multiphoton experiments the only way to avoid space-charge effects is to reduce the pressure as all molecules in the laser focal volume (about $100 \mu \mathrm{m}^{2}$ ) are ionized. In both types of experiments hydrocarbons are the worst offenders.

Despite experimental similarities, the dynamics of the single and multiphoton processes are quite different [1]. First, a single, high-energy photon couples to an inner-valence or core electron, while the multiphoton process affects, at least directly, the outermost electrons. Second, the single-photon ionization is well separated in time from the following fragmentation, while in the multiphoton case the two steps overlap in time forming a complex process of multielectron dissociative ionization (MEDI) [2].

\section{Covariance mapping technique}

The covariance mapping technique was first used to identify fragmentation channels of the MEDI process in intense sub-picosecond laser fields [3]. It relies on the fact that the pulse-topulse TOF spectra of the fragment ions have inherent statistical fluctuations. If one calcu- 
lates the covariance of every pair of TOF channels then the result can be displayed in the form of a map showing correlations of charge stage and kinetic energy between daughter fragment ions coming from a common parent molecule. The technique can be extended to study threefragment correlations [4] and correlations with photoelectrons [5].

In principle, the signal-to-noise ratio of covariance mapping does not deteriorate with increasing counting rate, even at the level when several ions are detected in the same channel of the TOF spectrum in each laser pulse. In practice, however, pulse-to-pulse fluctuations of the laser intensity forces one to reduce the counting rate (by lowering the sample pressure) to have on average no more than one ion in any TOF channel of interest per laser pulse. As the ion production always increase with the laser intensity, such fluctuations introduce extraneous correlation of every ion peak with every other one.

Extraneous correlations due to laser fluctuations can be removed using a statistical data processing technique [6]. It allows eomputation of an estimate, $\mathbf{C}(X, Y ; L)$, of the ion-ion covariance with the laser intensity held constant from the raw covariance map, $\mathbf{C}(X, Y)$, ion-laser covariance, $C(X, L)$, and laser variance, $V(L)$, using the following formula:

$$
\mathbf{C}(X, Y ; L)=\mathbf{C}(X, Y)-\frac{C(X, L) C(L, Y)}{V(L)}
$$

where $X=X(x)$ and $Y=Y(y)$ are ion detector amplitudes at TOFs $x$ and $y$, and $L$ is the laser photodetector amplitude (at TOF $=0$ ). A practical method of recording the necessary laser data is to combine the signals from the laser and ion detectors into one TOF spectrum. Now, if the covariance map is computed from the whole spectrum, then $C(X, L)$ and $C(L, Y)$ are, respectively, the map row and column at $\mathrm{TOF}=0$, and $V(L)=C(L, L)$ is the map element at both TOFs $=0$.

\section{Triple coincidence technique}

A (conceptual) simplification of covariance mapping is the triple coincidence technique, which applies [7] when the counting rate is low. In this case, processing of TOF spectra can be done more efficiently and it is possible to use a continuous source of excitation, such as synchrotron radiation.

High counting rate introduces a background of false coincidences on the coincidence map. This background can be removed using an analytical method [8] that is applicable when the counting rate is constant during the experimental run. However, it is not difficult to generalise this method to take into account a variable counting rate, for example, when the VUV radiation intensity drops with time due to loss of electrons in the synchrotron storage ring. The improved formula is

$$
\begin{aligned}
\mathbf{C}(x, y)=\mathbf{C}_{\text {raw }}(x, y)-\alpha \frac{w I}{T} & (V(x)+V(y)) \\
& +\left(2 \alpha^{2}+\beta\right)\left(\frac{w l}{T}\right)^{2} S
\end{aligned}
$$

where $\mathbf{C}(x, y)$ is the matrix of true coincidences, $x$ and $y$ are two TOF values, $\mathbf{C}_{\text {raw }}(x, y)$ is the matrix of raw data containing true and false coincidences, $w$ is the duration of the time-todigital converter window, $T$ is the experiment duration, $I$ is the total number of ions detected during the experiment, $V(x)$ and $V(y)$ are two values of the same TOF spectrum, and $S$ is the total number of ions detected during the experiment. The constants $\alpha$ and $\beta$ are given by the following formulae:

$$
\alpha=\frac{\left\langle R_{\mathrm{S}} R_{\mathrm{l}}\right\rangle}{\left\langle R_{\mathrm{S}}\right\rangle\left\langle R_{\mathrm{I}}\right\rangle}, \quad \beta=\frac{\left\langle R_{\mathrm{S}} R_{\mathrm{I}}^{2}\right\rangle}{\left\langle R_{\mathrm{S}}\right\rangle\left\langle R_{\mathrm{I}}\right\rangle^{2}}
$$

where $R_{\mathrm{S}}$ is the start rate, $R_{\mathrm{I}}$, is the ion rate, and the average, \langle\rangle , is taken over the experiment duration. 


\section{Site-selected dissociative ionization}

Single, high-energy photon can be used to create a hole in the core of a selected atom in a molecule. Selecting different atoms initiates different Auger processes that lead to different ionization and fragmentation channels. These channels were studied using soft $\mathrm{x}$-ray excitation and the triple coincidence technique combining threshold electron detection and ion TOF analysis [9]. These experiments are described in detail elsewhere in this volume [10].

\section{Multielectron dissociative ionization}

Tens or hundreds of low-energy photons combine to multiply ionize a molecule exposed to intense laser pulses. Subsequently, the molecule fragments due to strong Coulomb repulsion [11]. The divergence of the perturbation calculus at laser intensities exceeding $10^{14}$ $\mathrm{W} / \mathrm{cm}^{2}$ makes this process of multielectron dissociative ionization (MEDI) poorly understood.

A substantial insight into MEDI can be gained from studying atoms in intense laser fields. A suitable description here is the field ionization model. In this model the laser field depresses the atomic potential barrier until the outermost electron can tunnel out, and the first ionization occurs. As the second ionization potential is higher, the field has to increase before the second electron can tunnel out. This way, the electrons are removed one after another on the rising edge of the laser pulse. Experimental data on atoms fit this scenario well [12].

In molecules, however, the field ionization model, on its own, is insufficient to explain the kinetic energies of ions produced in MEDI. The ion-ion Coulomb repulsion in a multiply-ionized molecule allows one to estimate its dissociation timescale, giving typically a few tens of femtoseconds. This implies that with sub-picosecond laser pulses, the ionization and dissociation timescales overlap, i.e. the molecule dissociates while the electrons are being removed in the field ionization process. One consequence of this is that one would expect that longer laser pulses would produce lower ion kinetic energies; the molecule would dissociate more before reaching the high ionization stages making the Coulomb repulsion less effective. Experiments, however, show that the ion kinetic energies are independent of laser pulse length in the range $0.2-2$ ps [13]. Clearly, the field-ionization Coulomb explosion model needs to be improved.

Moreover, the dissociation energies are a certain fraction, $f$, of the Coulomb energy, $E=$ $Q_{1} Q_{2} / R_{\mathrm{e}}$, where $Q_{1}$ and $Q_{2}$ are the ion charges, and $R_{\mathrm{c}}$ is the equilibrium internuclear distance of the neutral molecule. It turns out that $f$ is molecule-specific (for example, $f=0.7$ for $\mathrm{I}_{2}$ and $f=0.45$ for $\mathrm{N}_{2}$ ).

One possible explanation is that the laser stabilises the molecule at a specific internuclear distance, induced by the laser field $[14,15]$. Alternatively, the ionization sequence in molecules could be much more rapid than in atoms [16, 17]. Agreement is achieved with experimental data if either of these processes occurs at a critical internuclear distance, $R_{\mathrm{C}}$, a factor $1 / f$ times larger than $R_{\mathrm{e}}$.

The ion kinetic energies and their appearance intensities can be predicted using a simple condition [15]: an electron is removed when the internuclear distance and the peak laser $E$ field are such that the electron energy level touches the top of, respectively, the inner (internuclear) and the outer potential barriers. This condition suggests a possible mechanism $[15,18]$ responsible for stabilization, as follows.

When the internuclear distance is smaller than $R_{\mathrm{C}}$, then the inner barrier is below the energy level and the electron can move freely between the two ions. The inter-ion free oscillation frequency of the electron is higher than the laser frequency. Therefore, the induced dipole 
moment, due to the electron hopping between the ions, is forced to oscillate in phase with the laser field making the down-field ion more positive than the up-field one. This charge asymmetry results in unequal forces exerted by the field on the ions, effectively producing a repulsive force on the nuclei, in addition to the Coulomb and chemical bond forces.

When the internuclear distance is larger than $R_{\mathrm{c}}$, then the inner barrier impedes the free movement of the electron, its free oscillation frequency becomes lower than the laser frequency, and the forced oscillation of the induced dipole moment is in antiphase with the laser field producing an attractive force on the nuclei. If the ionization stage of the molecule is not too large, then this attractive force (together with the force of chemical binding) may overcome the Coulomb repulsion and stabilise the molecular ion at an internuclear distance around $R_{\text {c. }}$.

A detailed analysis of MEDI in the Floquet theory, involving diagonalisation of molecular field-dressed states, is hampered by the complexity of the multielectron system. The repulsive and attractive forces due to the interaction of the induced dipole with the field are known in this formalism as bond softening [19, 20] and bond hardening [21, 22]. So far, this theory has been quite successful in describing the interaction of intense laser fields with a single-electron system, the hydrogen molecular ion.

When the internuclear distance passes $R_{\mathrm{c}}$ then the dipole-field phase is expected to change from 0 to $\pi$. At intermediate phases the oscillating electron can extract energy from the field. This energy transfer substantially enhances ionization near $R_{\mathrm{c}}$ making the ionization sequence more rapid than in atoms.

The same excitation is expected to produce VUV radiation [16]. The wavelength of this radiation is related to the dynamic Stark shift induced by the laser electric field. Figure 1 shows the electron potential wells at the critical

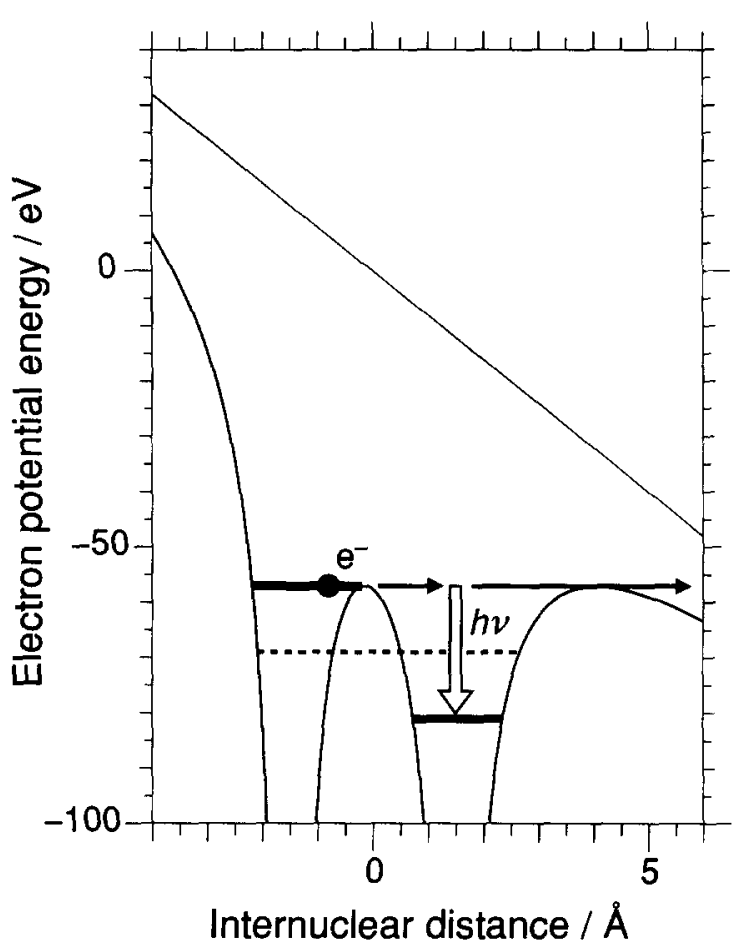

Figure 1. Stark-shift electron excitation. The excitation induces ionization (horizontal arrows) or VUV radiation (vertical arrow). The dashed line represents an unshifted electron energy level. The electron is in a double potential energy well produced by two $+3 e$ charges $6 \AA$ apart placed in an $8 \mathrm{~V} / \AA$ electric field. The system represents a 5 times ionized diatomic molecule with the internuclear distance relaxed to the critical value due to the action of the laser field.

internuclear distance, $R_{\mathrm{c}}$, modified by a crest of the laser field, $E_{\mathrm{L}}$. At a node, when the field drops to zero, the electron energy is the same in both wells (dashed line). When the field reaches a crest the Stark effect lifts the electron energy level in one well and lowers it in the other one (solid lines), making them differ by $E_{\mathrm{L}} R_{\mathrm{c}}$. Taking the typical values of $E_{\mathrm{L}}=10 \mathrm{~V} / \AA$ and $\mathrm{Rc}=3 \AA$, one can expect $30 \mathrm{eV}$ photons. We note that this, yet unobserved, mechanism is specific to molecules and different from the conventional high harmonic generation [23]. In the latter mechanism the electron is almost free, oscillates over a large distance outside an atom or molecule, and generates radiation when it 
collides with the core.

As the Stark-shift excitation can approach $100 \%$ efficiency, achieving population inversion may be contemplated. The main obstacle to constructing a VUV laser could be obtaining a sufficiently dense sample without too many photoelectrons screening the ions from the pumping laser field.

Having discussed the models of stabilisation and rapid ionization, a question remains which of them describes MEDI correctly. In our opinion, the most plausible explanation is that both are involved. Initially, when the laser intensity is low and the molecule is still neutral or in a low ionization stage, the internuclear distance stabilises at $R_{\mathrm{c}}$. As the intensity increases, the molecule experiences rapid ionization and starts to dissociate because Coulomb repulsion overcomes the stabilisation mechanism.

\section{References}

1. K Codling and L J Frasinski in Today and Tomorrow in Photoionisation ed. by $\mathrm{M}$ Ya Amusia and J B West (SERC Daresbury Laboratory, 1991) pp 72-77

2. L J Frasinski, K Codling, P A Hatherly, J Barr, I N Ross and W T Toner Phys. Rev. Lett. 582424 (1987)

3. L J Frasinski, K Codling and P A Hatherly Science 246 1029-1031 (1989)

4. L J Frasinski, P A Hatherly and K Codling Physics Letters A 156 227-232 (1991)

5. L J Frasinski, M Stankiewicz, P A Hatherly, G M Cross and K Codling Phys. Rev. A 46 R6789-R6792 (1992)

6. W J Krzanowski Principles of Multivariate Analysis Clarendon Press, Oxford (1990), section 14.4

7. L J Frasinski, M Stankiewicz, K J Randall, P A Hatherly and K Codling J. Phys. B: At. Mol. Phys. 19 L819-L824 (1986)

8. L J Frasinski, M Stankiewicz, P A Hatherly and $\mathrm{K}$ Codling Meas. Sci. Technol. 3 1188-1191 (1992)
9. P A Hatherly, K Codling, M Stankiewicz and M Roper J. Phys. B: At. Mol. Opt. Phys. 283249 (1995)

10. P A Hatherly, K Codling, M Stankiewicz and $\mathrm{M}$ Roper, submitted as Proceedings of the $11^{\text {th }}$ International Conference on Vacuum Ultraviolet Radiation Physics, special issue of Journal of Electron Spectroscopy and Related Phenomena (spring 1996)

11. K Codling and L J Frasinski Contemp. Phys. 35 243-255 (1994)

12. S Augst, D Strickland, D D Meyerhofer, S L Chin and J H Eberly Phys. Rev. Lett. 63 2212 (1989)

13. C Cornaggia, J Lavancier, D Normand, J Morellec, P Agostini, J P Chambaret and A Antonetti Phys. Rev. A 444499 (1991)

14. M Schmidt, D Normand and C Cornaggia Phys. Rev. A 50 5037-45 (1994)

15. J H Posthumus, L J Frasinski, A J Giles and K Codling J. Phys. B: At. Mol. Opt. Phys. 28 L349-L353 (1995)

16. K Codling, L J Frasinski and P A Hatherly J. Phys. B: At. Mol. Opt. Phys. 22 L321 (1989)

17. T Seideman, M Yu Ivanov and P B Corkum Phys. Rev. Lett. (submitted 1995)

18. J H Posthumus, A J Giles, M R Thompson, $\mathrm{L} \mathrm{J}$ Frasinski and $\mathrm{K}$ Codling in Proceedings of the NATO Workshop on Super Intense Lasers in Atomic Physics, Moscow (4-9 August 1995)

19. A D Bandrauk and M L Sink J. Chem. Phys. 741110 (1981)

20. P H Bucksbaum, A Zavriyev, H G Muller and D W Schumacher Phys. Rev. Lett. 64 1883 (1990)

21. A Giusti-Suzor and F H Mies Phys. Rev. Lett. 683869 (1992)

22. A Zavriyev, P H Bucksbaum, J Squier and F Saline Phys. Rev. Lett. 701077 (1993)

23. Y Liang, S Augst, S L Chin, Y Beaudoin and M Chaker J. Phys. B: At. Mol. Opt. Phys. 27 5119-30 (1994) 